Algebra univers. 54 (2005) 1-22

$0002-5240 / 05 / 010001-22$

DOI $10.1007 / \mathrm{s} 00012-005-1918-0$

(C) Birkhäuser Verlag, Basel, 2005

Algebra Universalis

\title{
Full does not imply strong, does it?
}

\author{
Brian A. Davey, Miroslav Haviar, and Ross Willard
}

\begin{abstract}
We give a duality for the variety of bounded distributive lattices that is not full (and therefore not strong) although it is full but not strong at the finite level. While this does not give a complete solution to the "Full vs Strong" Problem, which dates back to the beginnings of natural duality theory in 1980, it does solve it at the finite level. One consequence of this result is that although there is a Duality Compactness Theorem, which says that if an alter ego of finite type yields a duality at the finite level then it yields a duality, there cannot be a corresponding Full Duality Compactness Theorem.
\end{abstract}

Is every full duality strong? This question is as old as the theory of natural dualities, and remains one of the most tantalising open problems in the foundations of the theory. A full duality is a special kind of dual equivalence between a quasi-variety $\mathcal{A}:=\mathbb{I S P}(\underline{\mathbf{M}})$ of algebras and a category $\boldsymbol{X}:=\mathbb{I S}_{\mathrm{C}} \mathbb{P}^{+}(\underset{\sim}{\mathbf{M}})$ of structured topological spaces. The earliest version of the "Full versus Strong" problem was formulated by Davey and Werner [13]:

Question 1. If $\underset{\sim}{\mathbf{M}}$ yields a full duality between $\mathcal{A}:=\mathbb{I S P}(\underline{\mathbf{M}})$ and $\boldsymbol{X}:=\mathbb{I S}_{\mathrm{c}} \mathbb{P}^{+}(\stackrel{\mathbf{M}}{\sim})$ does it follow that $\underset{\sim}{\mathbf{M}}$ is injective in $\boldsymbol{X}$ ?

This question stems from a fundamental asymmetry in all known full dualities. For every full duality between $\mathcal{A}:=\mathbb{I S P}(\underline{\mathbf{M}})$ and $\boldsymbol{X}:=\mathbb{I S}_{\mathrm{c}} \mathbb{P}^{+}(\underset{\sim}{\mathbf{M}})$, the injectivity of the algebra $\underline{\mathbf{M}}$ in $\mathcal{A}$ implies the injectivity of the alter ego $\underline{\mathbf{M}}$ in $\mathcal{X}$. But the converse statement is false. Nevertheless, $\underset{\sim}{\mathbf{M}}$ is injective in $\boldsymbol{X}$ in every known example of a full duality. (The interconnections between the injectivity of $\underline{\mathbf{M}}$ and the injectivity of $\underset{\sim}{\mathbf{M}}$ were discussed at length in [13]: see Proposition 1.11 on page 128 and pages 258-263 in the Appendix. The setting there was a general category-theoretic one and not specific to natural dualities. Some refinements in the setting of natural dualities are given in Exercises 6.2-6.6 of Clark and Davey [2].) The monograph [2] is recommended as the best source of basic facts as well as recent developments in the theory of natural dualities.

Clark and Krauss [4] introduced the important notions of term-closed subsets of $M^{S}$ and hom-closed subsets of $M^{S}$ and proved that they are the same. They

Presented by R. W. Quackenbush.

Received October 1, 2002; accepted in final form November 10, 2004.

2000 Mathematics Subject Classification: 06D50; 08C05, 08C15, 18A40, 08A55.

Key words and phrases: Natural duality, full duality, strong duality, distributive lattices.

The authors wish to thank Jane Pitkethly for her many helpful comments on the first draft of this paper. The second author was supported by a La Trobe University Fellowship. 
showed that, if $\underset{\sim}{\mathbf{M}}$ yields a duality on $\mathcal{A}$ and every closed substructure of a nonzero power of $\underset{\sim}{\mathbf{M}}$ is term-closed, then $\underset{\sim}{\mathbf{M}}$ yields a full duality on $\mathcal{A}$. Moreover, they observed that every natural duality known at that time could be proved to be full by establishing this apparently stronger condition. This led to the following (equivalent) problems:

Question 2. If $\underset{\sim}{\mathbf{M}}$ yields a full duality between $\mathcal{A}:=\mathbb{I S P}(\underline{\mathbf{M}})$ and $\boldsymbol{X}:=\mathbb{I} \mathbb{S}_{\mathrm{C}} \mathbb{P}^{+}(\underset{\sim}{\mathbf{M}})$ does it follow that every closed substructure of a non-zero power of $\underset{\sim}{\mathbf{M}}$ is termclosed?

Question 3. If $\underset{\sim}{\mathbf{M}}$ yields a full duality between $\mathcal{A}:=\mathbb{I S P}(\underline{\mathbf{M}})$ and $\boldsymbol{X}:=\mathbb{\mathbb { S } _ { \mathrm { c } }} \mathbb{P}^{+}(\underset{\sim}{\mathbf{M}})$ does it follow that every closed substructure of a non-zero power of $\underset{\sim}{\mathbf{M}}$ is homclosed?

Later, Clark and Davey [1] showed that the questions posed in [13] and [4] were one and the same. They proved that, given a duality between $\mathcal{A}:=\mathbb{I S P}(\underline{\mathbf{M}})$ and $\boldsymbol{X}:=\mathbb{\mathbb { S } _ { \mathrm { c } }} \mathbb{P}^{+}(\underset{\sim}{\mathbf{M}})$, every closed substructure of a non-zero power of $\underset{\sim}{\mathbf{M}}$ is term-closed (hom-closed) if and only if $\underset{\sim}{\mathbf{M}}$ yields a full duality on $\mathcal{A}$ and $\underset{\sim}{\mathbf{M}}$ is injective in $\boldsymbol{X}$. When these equivalent conditions hold we now say that $\underset{\sim}{\mathbf{M}}$ yields a strong duality on $\mathcal{A}$. We refer the reader to the text by Clark and Davey [2] for the definitions of term-closed and hom-closed subsets of $M^{S}$ and for a detailed discussion of full and strong natural dualities.

All three problems above can now be re-formulated as the following question.

Question. Is every full duality strong?

In this paper we give a partial answer to this question by constructing a duality for the quasi-variety of bounded distributive lattices, based on the three-element chain, that is full but not strong at the finite level. We show that our duality is not even full, and therefore not strong, at the infinite level. (See Theorem 1.) This means there is no Full Duality Compactness Theorem - if an alter ego of finite type yields a full duality at the finite level, then it does not follow that it yields a full duality. We also prove that any duality for the quasi-variety of bounded distributive lattices, based on the three-element chain, that is full is necessarily strong. (See Theorem 2.)

At the beginning of this story was a failed attempt by the first and third authors to prove that full implies strong. Within days of distributing a preprint of a "proof", they discovered a gaping hole and withdrew their claim. Nevertheless, all was not lost. With a lot of further work (and further failed attempts), the present team of three was able to obtain the results presented in this paper and its companion, [10].

In the first section of this paper, we shall introduce and state our theorems. In Section 2, we list the quasi-equations upon which many of our arguments are based. Our first main theorem is proved in Section 3. In Section 4, we give a sneak preview 
of structural entailment, which will be developed more fully in [10]. Just enough is presented here to facilitate the proof of our second main theorem in Section 5 . In the final section, we give descriptions of the three dual categories which arise in our work.

\section{Full versus strong}

Davey, Haviar and Priestley [8] proved that the three-element bounded distributive lattice

$$
\underline{\mathbf{M}}=\langle\{0, a, 1\} ; \vee, \wedge, 0,1\rangle
$$

is endodualisable. (See also Davey [6] and Davey and Pitkethly [11].) The only non-identity endomorphisms of $\underline{\mathbf{M}}$ are $f$ and $g$, given by $f(0)=f(a)=0, f(1)=1$ and $g(0)=0, g(a)=g(1)=1$. So it follows that $\underline{\mathbf{M}}$ is dualised by the alter ego

$$
\underset{\sim}{\sim}=\langle\{0, a, 1\} ; f, g, \mathcal{T}\rangle \text {. }
$$

This duality is certainly not strong: for example, the set $\{0,1\}$ determines a substructure of $\underset{\sim}{\mathbf{M}}$ but it is not hom-closed as it is not closed under the binary algebraic partial operation $m:\{(0,0),(0,1),(1,1)\} \rightarrow\{0, a, 1\}$ with $m(0,0)=0, m(0,1)=a$ and $m(1,1)=1$ (see Figure 1). To see that the duality is not full requires a larger structure: a simple calculation shows that $X:=\{(0,0),(0, a),(0,1),(a, 1),(1,1)\}$ determines a substructure of ${\underset{\mathbf{M}}{ }}^{2}$, that $\mathrm{E}(\mathbf{X})$ is a four-element chain and that $|\operatorname{DE}(\mathbf{X})|=6$, whence $\mathbf{X} \nsucceq \operatorname{DE}(\mathbf{X})$.
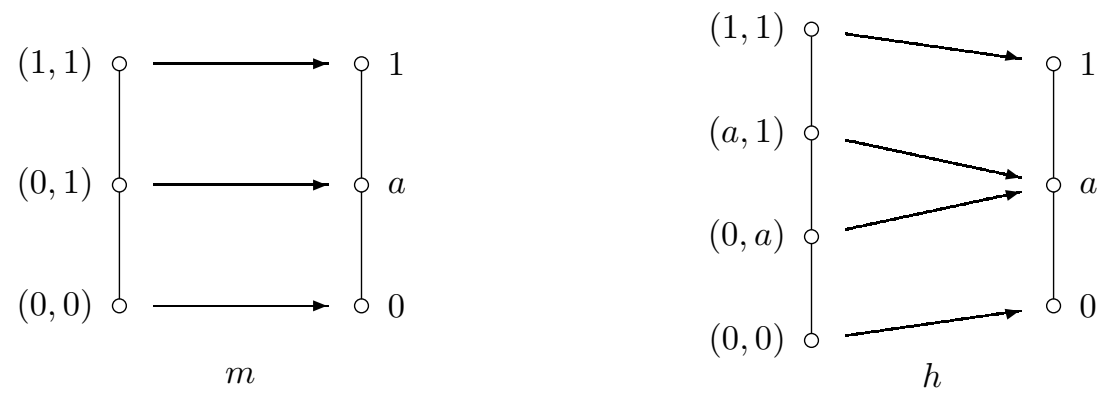

Figure 1. The partial operations $m$ and $h$

This paper concerns the enrichment of $\underset{\sim}{\mathbf{M}}$ given by the alter ego

$$
\mathbf{M}_{h}=\langle\{0, a, 1\} ; f, g, h, \mathcal{T}\rangle,
$$

where the partial binary operation $h:\{(0,0),(0, a),(a, 1),(1,1)\} \rightarrow\{0, a, 1\}$ is defined by $h(0,0)=0, h(0, a)=h(a, 1)=a$ and $h(1,1)=1$ (see Figure 1). We shall 
see that this structure yields a duality which is not full, although it is full at the finite level. This duality is certainly not strong (at the finite level): again, the set $\{0,1\}$ determines a substructure of $\mathbf{M}_{h}$ but is not closed under $m$. The partial operation $m$, while not in the type of $\mathbf{M}_{h}$, will play a vital role in our deliberations. By the main result of Davey and Haviar [7] (see also [9], where the original computer-based proof was given), the alter ego

$$
\mathbb{M}_{m}=\langle\{0, a, 1\} ; f, g, m, \mathcal{T}\rangle
$$

does yield a strong and therefore full duality. As we shall see in Lemma 2.2, the structure $\underset{\sim}{\mathbf{M}}$ may be viewed as intermediate between $\underset{\sim}{\mathbf{M}}$ and $\underset{\sim}{\mathbf{M}}$.

Theorem 1. The structure $\mathbb{\mathbf { M }}_{h}=\langle\{0, a, 1\} ; f, g, h, \mathcal{T}\rangle$ yields a full duality, which is not strong, on the category $\mathcal{D}_{\text {fin }}$ of finite bounded distributive lattices and yields a duality which is not even full on the category $\mathcal{D}$ of all bounded distributive lattices.

In view of our success at the finite level, we could not be blamed for hoping that, with a bit more effort, we might find an example of a full but not strong duality for $\mathcal{D}$ based on the three-element chain. It turned out that this hope was forlorn.

Theorem 2. Every full duality for the category $\mathcal{D}$ of all bounded distributive lattices that is based on the three-element chain is strong.

\section{A clutch of quasi-equations}

Before we embark on the proofs of the theorems, we make a short excursion into the three dual categories whose subtle interplay is at the heart of our analysis.

Many of our arguments rely only on certain equations and quasi-equations which hold in the structures $\underset{\sim}{\mathbf{M}}, \underset{\sim}{\underset{\sim}{\mathbf{M}}}$ and $\underset{\sim}{\mathbf{M}}{ }_{m}$. We collect them together here for future reference. Since equations and quasi-equations involving partial operations may be somewhat unfamiliar, we note that, for a partial binary operation $p$, the statement $(x, y) \in \operatorname{dom}(p)$ can be expressed by the equation $p(x, y)=p(x, y)$. Each of the following is equivalent to a finite conjunct of quasi-equations:

(1) $g(f(x))=f(f(x))=f(x)$ and $f(g(x))=g(g(x))=g(x)$,

(2) $f(x)=x \Longleftrightarrow g(x)=x$, that is, $\operatorname{fix}(f)=\operatorname{fix}(g)$,

(3) $[f(x)=g(y) \& f(y)=g(x)] \Longrightarrow x=y$,

(4) $[f(x)=f(y) \& g(x)=g(y)] \Longrightarrow x=y$,

(5) $(x, y) \in \operatorname{dom}(h) \Longleftrightarrow g(x)=f(y)$,

(6) $(x, y) \in \operatorname{dom}(h) \Longrightarrow f(h(x, y))=f(x) \& g(h(x, y))=g(y)$,

(7) $(f(x), g(x)) \in \operatorname{dom}(m)$ and $m(f(x), g(x))=x$,

(8) $(x, y) \in \operatorname{dom}(m) \Longrightarrow f(m(x, y))=x \quad \& g(m(x, y))=y$,

(9) $(x, y) \in \operatorname{dom}(m) \&(y, z) \in \operatorname{dom}(m) \Longrightarrow(x, z) \in \operatorname{dom}(m)$, 
(10) $(x, x) \in \operatorname{dom}(m) \Longrightarrow f(x)=x \& g(x)=x$,

(11) $(x, x) \in \operatorname{dom}(m) \Longrightarrow m(x, x)=x$,

(12) $(x, y) \in \operatorname{dom}(h) \Longrightarrow(f(x), g(y)) \in \operatorname{dom}(m) \& h(x, y)=m(f(x), g(y))$.

These quasi-equations are certainly not independent.

Lemma 2.1. The following relations hold amongst the quasi-equations listed above:

(a) (1) $\Longrightarrow(2)$;

(b) $(7) \Longrightarrow(4)$;

(c) given (4) and (8), we have (10) $\Longleftrightarrow(11)$;

(d) $[(5) \&(6) \&(7) \&(8) \&(9)] \Longrightarrow(12)$.

Proof. (a) and (b) are almost trivial, (c) is easy while (d) requires a little explanation. Assume that (5)-(9) hold and assume that $(x, y) \in \operatorname{dom}(h)$. By $(5)$, we have $g(x)=f(y)$ and, by $(7)$, we have $(f(x), g(x)) \in \operatorname{dom}(m)$ and $(f(y), g(y)) \in \operatorname{dom}(m)$. Hence, by $(9),(f(x), g(y)) \in \operatorname{dom}(m)$. By (6) and (8), we have

$$
f(h(x, y))=f(x)=f(m(f(x), g(y))) \text { and } g(h(x, y))=g(y)=g(m(f(x), g(y))) .
$$

Since (7) implies (4), we may apply (4) to conclude that $h(x, y)=m(f(x), g(y))$. Hence (d) holds.

We now introduce topological quasi-equational classes corresponding to each of the topological quasi-varieties

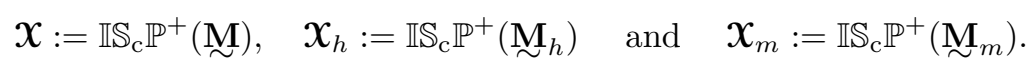

Let $G$ be a set of operation symbols, $H$ a set of partial-operation symbols and $\Sigma$ a set of equations and quasi-equations of type $G \cup H$. Then $\operatorname{Mod}_{\mathcal{T}}(\Sigma)$ denotes the class of all Boolean topological models of $\Sigma$, that is, structures $\mathbf{X}=\left\langle X ; G^{\mathbf{X}}, H^{\mathbf{X}}, \mathcal{T}\right\rangle$ such that $\mathcal{T}$ is a Boolean topology on $X$, for each $g \in G$ the corresponding operation $g^{\mathbf{X}}$ on $X$ is continuous, for each $h \in H$ the corresponding partial operation $h^{\mathbf{X}}$ on $X$ has a (topologically) closed domain and is continuous, and all of the quasiequations in $\Sigma$ hold in $\mathbf{X}$. Whenever possible, we drop the superscript $\mathbf{X}$. (In general, a set $R$ of relation symbols is allowed as well.) Now consider the following sets of quasi-equations

$$
\begin{aligned}
& \Sigma:=\{(1),(2),(3),(4)\}, \\
& \Sigma_{h}:=\{(1),(2),(3),(4),(5),(6)\}, \\
& \Sigma_{m}:=\{(1),(2),(3),(4),(7),(8),(9),(10),(11)\},
\end{aligned}
$$

and note that $\underset{\sim}{\mathbf{M}} \models \Sigma,{\underset{\sim}{\sim}}_{h}=\Sigma_{h}$ and $\underset{\sim}{\mathbf{M}_{m}}=\Sigma_{m}$. Define $\boldsymbol{y}, \boldsymbol{y}_{h}$ and $\boldsymbol{y}_{m}$ to be the categories with $\operatorname{Mod}_{\mathcal{T}}(\Sigma), \operatorname{Mod}_{\mathcal{T}}\left(\Sigma_{h}\right)$, and $\operatorname{Mod}_{\mathcal{T}}\left(\Sigma_{m}\right)$, respectively, as their classes of objects and with all continuous homomorphisms between objects as morphisms.

There is an obvious forgetful functor from $\boldsymbol{y}_{h}$ into $\boldsymbol{y}$ : just drop the partial operation $h$. The following lemma, whose proof is easy and left to the reader, 
shows that there is a natural (but not so obvious) forgetful functor from $\boldsymbol{y}_{m}$ into $\boldsymbol{y}_{h}$ : define $h$ in terms of $f, g$ and $m$, then forget $m$ and remember $h$. This justifies our earlier claim that $\underset{\sim}{\mathbf{M}_{h}}$ is an intermediate structure between $\underset{\sim}{\mathbf{M}}$ and $\underset{\sim}{\mathbf{M}} \boldsymbol{m}$.

Lemma 2.2. Let $\langle X ; f, g, m, \mathcal{T}\rangle$ belong to $\boldsymbol{y}_{m}$ and define a partial operation $h$ on $X$ by $\operatorname{dom}(h)=\left\{(x, y) \in X^{2} \mid g(x)=f(y)\right\}$ and $h(x, y):=m(f(x), g(y))$, for all $(x, y) \in \operatorname{dom}(h)$. Then $\langle X ; f, g, h, \mathcal{T}\rangle$ belongs to $\boldsymbol{y}_{h}$.

If we suppress the forgetful functors, we obtain the following diagram of (nonfull) subcategories.

$$
\begin{aligned}
\boldsymbol{y}_{m} & \subseteq \boldsymbol{y}_{h} \subseteq \boldsymbol{y} \\
\cup \mathrm{I} & \cup \mathrm{U} \\
\boldsymbol{x}_{m} & \subseteq \boldsymbol{x}_{h} \subseteq \boldsymbol{x}
\end{aligned}
$$

We turn now to the proof of Theorem 1 . Wherever possible we shall work within the appropriate category $\boldsymbol{y}, \boldsymbol{y}_{h}$ or $\boldsymbol{y}_{m}$. We shall restrict our attention to objects in the subcategory $\boldsymbol{X}, \boldsymbol{x}_{h}$ or $\boldsymbol{x}_{m}$ only when forced to.

\section{The proof of Theorem 1}

The idea behind the proof of Theorem 1 is quite simple. We shall be working with two different dualities for the category $\mathcal{D}$ of bounded distributive lattices. Priestley's duality is based on the two-element bounded lattice $\underline{\mathbf{D}}=\langle\{0,1\} ; \vee, \wedge, 0,1\rangle$ and the discrete ordered space $\underset{\sim}{\mathbf{D}}=\langle\{0,1\} ; \leqslant, \mathcal{T}\rangle$. Let

$$
\mathrm{H}: \mathcal{D} \rightarrow \mathcal{P} \text { and } \mathrm{K}: \mathcal{P} \rightarrow \mathcal{D}
$$

be the associated functors, where $\mathcal{P}:=\mathbb{I S}_{\mathrm{c}} \mathbb{P}^{+}(\underset{\sim}{\mathbf{D}})$ is the category of Priestley spaces. (For details on Priestley duality see Priestley [14], Davey and Priestley [12] and Clark and Davey [2].) We will also be using the duality for $\mathcal{D}$ based on $\underline{\mathbf{M}}$ and ${\underset{\mathbf{M}}{\sim}}_{h}$ given by functors

$$
\mathrm{D}: \mathcal{D} \rightarrow \boldsymbol{X}_{h} \text { and } \mathrm{E}: \boldsymbol{X}_{h} \rightarrow \mathcal{D} .
$$

The functors $\mathrm{H}, \mathrm{K}, \mathrm{D}$ and $\mathrm{E}$ are hom-functors and are given, at the underlying-set level on objects, by $\mathrm{H}(\mathbf{A})=\mathcal{D}(\mathbf{A}, \underline{\mathbf{D}}), \mathrm{K}(\mathbf{Y})=\mathcal{P}(\mathbf{Y}, \underline{\mathbf{D}}), \mathrm{D}(\mathbf{A})=\mathcal{D}(\mathbf{A}, \underline{\mathbf{M}})$ and $\mathrm{E}(\mathbf{X})=\boldsymbol{X}_{h}\left(\mathbf{X}, \mathbf{M}_{h}\right)$, for all $\mathbf{A} \in \mathcal{D}$, all $\mathbf{Y} \in \mathcal{P}$ and all $\mathbf{X} \in \boldsymbol{X}_{h}$.

Consider a lattice $\mathbf{A} \in \mathcal{D}$. We can easily recover the Priestley dual $\mathrm{H}(\mathbf{A})$ from the dual $\mathrm{D}(\mathbf{A})$. The underlying set of $\mathrm{H}(\mathbf{A})$ is $\mathcal{D}(\mathbf{A}, \underline{\mathbf{D}})$, which is equal to the fixpoint set $\operatorname{fix}(f) \subseteq \mathcal{D}(\mathbf{A}, \underline{\mathbf{M}})$. The maps in $\mathrm{H}(\mathbf{A})$ are ordered pointwise using the order $0 \leqslant 1$ on $D$. It is straightforward to check that, for all $u, v \in \mathcal{D}(\mathbf{A}, \underline{\mathbf{D}})$, we have $u \leqslant v$ if and only if there is $x \in \mathcal{D}(\mathbf{A}, \underline{\mathbf{M}})$ with $f \circ x=u$ and $g \circ x=v$. We begin by mimicking this in an arbitrary structure $\mathbf{X} \in \mathbf{y}$. 
Let $\mathbf{X} \in \mathbf{y}$ and define $P_{\mathbf{X}}:=\operatorname{fix}(f)=\operatorname{fix}(g) \subseteq X$. Endow $P_{\mathbf{X}}$ with the subspace topology and define a binary relation $\preccurlyeq$ on $P_{\mathbf{X}}$ by

$$
u \preccurlyeq v \Longleftrightarrow(\exists x \in X) f(x)=u \quad \& \quad g(x)=v .
$$

We shall show that, for all $\mathbf{X} \in \mathbf{y}_{h}$, the structure $\mathbf{P}_{\mathbf{X}}:=\left\langle P_{\mathbf{X}} ; \preccurlyeq, \mathcal{T}\right\rangle$ is an ordered Boolean space. Note that $\mathbf{P}_{\mathrm{D}(\mathbf{A})}=\mathrm{H}(\mathbf{A})$, for all $\mathbf{A} \in \mathcal{D}$. If $\mathbf{P}_{\mathbf{X}}$ is a Priestley space and in particular if $\mathbf{X}$ is finite, then, by Priestley duality, $\mathbf{P}_{\mathbf{X}} \cong \operatorname{HK}\left(\mathbf{P}_{\mathbf{X}}\right)$. Using this fact, we are able to prove that, for each $\mathbf{X} \in \boldsymbol{X}_{h}$, we have $\mathbf{X} \cong \operatorname{DE}(\mathbf{X})$ if and only if $\mathbf{P}_{\mathbf{X}}$ is a Priestley space. Finally, we present an example of a (necessarily infinite) structure $\mathbf{X}$ in $\boldsymbol{X}_{h}$ for which $\mathbf{P}_{\mathbf{X}}$ is not a Priestley space.

Lemma 3.1. Let $\mathbf{X} \in \mathbf{y}$ and define $\mathbf{P}_{\mathbf{X}}:=\left\langle P_{\mathbf{X}} ; \preccurlyeq, \mathcal{T}\right\rangle$ as above.

(a) The topological space $\left\langle P_{\mathbf{X}} ; \mathcal{T}\right\rangle$ is compact and therefore Boolean.

(b) The relation $\preccurlyeq$ is topologically closed.

(c) The relation $\preccurlyeq$ is reflexive and anti-symmetric.

(d) The relation $\preccurlyeq$ is transitive, and therefore an order, provided $\mathbf{X}$ is the reduct of a structure in $\boldsymbol{y}_{h}$.

Proof. Since the topology on $X$ is Hausdorff and $f$ is continuous, $P_{\mathbf{X}}=f \operatorname{fix}(f)$ is closed in $X$ and hence, since $X$ is compact, so is $P_{\mathbf{X}}$. Note that

$$
\preccurlyeq=(f \sqcap g)(X)=\{(f(x), g(x)) \mid x \in X\},
$$

where $f \sqcap g: X \rightarrow X \times X$ is the natural map. Thus, since the topology on $X$ is compact and Hausdorff and since $f \sqcap g$ is continuous, the set $\preccurlyeq$ is closed in $X \times X$ and is therefore closed in $P_{\mathbf{X}} \times P_{\mathbf{X}}$. This proves (a) and (b).

Let $u \in P_{\mathbf{X}}$. By $(2), f(u)=u=g(u)$, whence $u \preccurlyeq u$. Hence, the relation $\preccurlyeq$ is reflexive. Now let $u, v \in P_{\mathbf{X}}$ with $u \preccurlyeq v$ and $v \preccurlyeq u$. There exist $x, y \in X$ with $f(x)=u=g(y)$ and $g(x)=v=f(y)$. By (3), we have $x=y$ and consequently $u=f(x)=f(y)=v$. Hence, the relation $\preccurlyeq$ is reflexive and anti-symmetric, which establishes (c).

Finally, assume that $\mathbf{X}$ is the reduct of a structure in $\boldsymbol{y}_{h}$. Let $u, v, w \in P_{\mathbf{X}}$ with $u \preccurlyeq v \preccurlyeq w$. There exist $x, y \in X$ with $f(x)=u, g(x)=v=f(y)$ and $g(y)=w$. By (5) and (6), we have $z:=h(x, y)$ in $X$ with $f(z)=u$ and $g(z)=w$, that is, $u \preccurlyeq w$. Hence, the relation $\preccurlyeq$ is transitive, which proves $(\mathrm{d})$.

Let $\mathbf{X} \in \boldsymbol{X}_{h}$. Since $\boldsymbol{X}_{h}=\mathbb{I S}_{\mathrm{C}} \mathbb{P}^{+}\left(\underset{\sim}{\mathbf{M}_{h}}\right)$, the natural evaluation map $\varepsilon_{\mathbf{X}}: \mathbf{X} \rightarrow$ $\mathrm{DE}(\mathbf{X})$ is an embedding, and by the lemma above, since $\boldsymbol{X}_{h} \subseteq \boldsymbol{y}_{h}$, the structure $\mathbf{P}_{\mathbf{X}}$ is an ordered Boolean space. As remarked earlier, since every finite ordered Boolean space is discrete and is therefore a Priestley space, the first half of Theorem 1 follows at once from our next result. (Note that we have extended the domain of the functor E and the codomain of the functor D from $\boldsymbol{x}_{h}$ to $\boldsymbol{y}_{h}$.) 
Proposition 3.2. Let $\mathrm{D}: \mathcal{D} \rightarrow \boldsymbol{y}_{h}$ and $\mathrm{E}: \boldsymbol{y}_{h} \rightarrow \mathcal{D}$ be the hom-functors induced by the alter ego $\mathbf{M}_{h}$.

(a) If $\mathbf{X} \in \mathbf{y}_{h}$ and the ordered Boolean space $\mathbf{P}_{\mathbf{X}}$ is a Priestley space, then the evaluation map $\varepsilon_{\mathbf{X}}: \mathbf{X} \rightarrow \operatorname{DE}(\mathbf{X})$ is surjective.

(b) Let $\mathbf{X} \in \mathbf{y}_{h}$. Then $\varepsilon_{\mathbf{X}}: \mathbf{X} \rightarrow \mathrm{DE}(\mathbf{X})$ is an isomorphism if and only if $\mathbf{X} \in \mathbf{X}_{h}$ and $\mathbf{P}_{\mathbf{X}}$ is a Priestley space.

Proof. Let $\mathbf{X} \in \boldsymbol{y}_{h}$. We first show that (b) is an easy consequence of (a). If $\mathbf{X} \cong \mathrm{DE}(\mathbf{X})=\mathrm{D}(\mathbf{A})$, with $\mathbf{A}:=\mathrm{E}(\mathbf{X})$, then $\mathbf{X} \in \boldsymbol{X}_{h}$ and the ordered space $\mathbf{P}_{\mathbf{X}}$ is isomorphic to $\mathrm{H}(\mathbf{A})$, viewed as the subset fix $(f)$ of $\mathrm{D}(\mathbf{A})$, and consequently $\mathbf{P}_{\mathbf{X}}$ is a Priestley space since $\mathrm{H}(\mathbf{A})$ is. The converse follows at once from (a). As we shall now see, the proof of (a) requires considerably more effort.

Assume that $\mathbf{P}_{\mathbf{X}}$ is a Priestley space. First of all, we shall prove that the bounded distributive lattices $\mathrm{E}(\mathbf{X})$, with underlying set $\boldsymbol{y}_{h}\left(\mathbf{X}, \mathbf{M}_{h}\right)$, and $\mathrm{K}\left(\mathbf{P}_{\mathbf{X}}\right)$, with underlying set $\mathcal{P}\left(\mathbf{P}_{\mathbf{X}}, \underset{\sim}{\mathbf{D}}\right)$, are isomorphic. For each $\alpha \in \mathbf{y}_{h}\left(\mathbf{X}, \mathbf{M}_{h}\right)$, define a map $\alpha^{*}:=\alpha \uparrow_{P_{\mathbf{X}}}: P_{\mathbf{X}} \rightarrow D=\{0,1\}$. Then $\alpha^{*}$ is well defined since $\alpha$ preserves $f$. Now let $\alpha \in \boldsymbol{y}_{h}\left(\mathbf{X}, \mathbf{M}_{h}\right)$. Since $\alpha$ is continuous, so is $\alpha^{*}$. Moreover, for all $u, v \in P_{\mathbf{X}}$,

$$
\begin{aligned}
u \preccurlyeq v & \Longleftrightarrow(\exists x \in X) f(x)=u \& g(x)=v \\
& \Longrightarrow \alpha^{*}(u)=\alpha(f(x))=f(\alpha(x)) \& \alpha^{*}(v)=\alpha(g(x))=g(\alpha(x)) \\
& \Longrightarrow \alpha^{*}(u) \leqslant \alpha^{*}(v) .
\end{aligned}
$$

Hence, $\alpha^{*} \in \mathcal{P}\left(\mathbf{P}_{\mathbf{X}}, \underset{\sim}{\mathbf{D}}\right)$. For each $\varphi \in \mathcal{P}\left(\mathbf{P}_{\mathbf{X}}, \underset{\sim}{\mathbf{D}}\right)$, define $\varphi^{*}: X \rightarrow M$ by

$$
\varphi^{*}(x):=m(\varphi(f(x)), \varphi(g(x)))
$$

for all $x \in X$. Now let $\varphi \in \mathcal{P}\left(\mathbf{P}_{\mathbf{X}}, \underset{\sim}{\mathbf{D}}\right)$. Since $\varphi$ is order-preserving, since $f(x) \preccurlyeq g(x)$ in $\mathbf{P}_{\mathbf{X}}$, for all $x \in X$, and since the domain of $m$ is precisely $\leqslant \subseteq D^{2} \subseteq M^{2}$, it follows that $\varphi^{*}$ is well defined. We want to show that $\varphi^{*} \in \mathbf{y}_{h}\left(\mathbf{X}, \mathbf{M}_{h}\right)$. Since the maps $\varphi, f$ and $g$ are continuous, so is $\varphi^{*}$. For all $x \in X$,

$$
\begin{aligned}
\varphi^{*}(f(x)) & =m(\varphi(f(f(x))), \varphi(g(f(x)))) & & \text { definition of } \varphi^{*} \\
& =m(\varphi(f(x)), \varphi(f(x))) & & \text { as } \mathbf{X} \models(1) \\
& =\varphi(f(x)) & & \text { by (11) in } M \\
& =f(m(\varphi(f(x)), \varphi(g(x)))) & & \text { by (8) in } M \\
& =f\left(\varphi^{*}(x)\right) & & \text { definition of } \varphi^{*} .
\end{aligned}
$$

Thus $\varphi^{*}$ preserves $f$ and, by symmetry, $\varphi^{*}$ also preserves $g$. Let $(x, y) \in \operatorname{dom}(h)$. Since $\varphi^{*}$ preserves $f$ and $g$, we have

$$
g\left(\varphi^{*}(x)\right)=\varphi^{*}(g(x))=\varphi^{*}(f(y))=f\left(\varphi^{*}(y)\right) \quad \text { as } \mathbf{X} \models(5) .
$$


Hence $\left(\varphi^{*}(x), \varphi^{*}(y)\right) \in \operatorname{dom}(h)$ in $M$, by $(5)$. But,

$$
\begin{aligned}
h\left(\varphi^{*}(x)\right. & \left., \varphi^{*}(y)\right) & & \\
& =h(m(\varphi(f(x)), \varphi(g(x))), m(\varphi(f(y)), \varphi(g(y)))) & & \text { definition of } \varphi^{*} \\
& =m(f(m(\varphi(f(x)), \varphi(g(x)))), g(m(\varphi(f(y)), \varphi(g(y))))) & & \text { by }(12) \text { in } M \\
& =m(\varphi(f(x)), \varphi(g(y))) & & \text { by }(8) \text { in } M \\
& =m(\varphi(f(h(x, y))), \varphi(g(h(x, y)))) & & \text { as } \mathbf{X}=(6) \\
& =\varphi^{*}(h(x, y)) & & \text { definition of } \varphi^{*} .
\end{aligned}
$$

So $\varphi^{*}$ preserves $h$, and consequently $\varphi^{*} \in \boldsymbol{y}_{h}\left(\mathbf{X}, \mathbf{M}_{h}\right)$.

If $\alpha \leqslant \beta$ in $\boldsymbol{y}_{h}\left(\mathbf{X},{\underset{\sim}{\sim}}_{h}\right)$, then certainly $\alpha^{*}=\left.\alpha\right|_{P \mathbf{X}} \leqslant\left.\beta\right|_{P \mathbf{X}}=\beta^{*}$ in $\mathcal{P}\left(\mathbf{P}_{\mathbf{X}}, \mathbf{D}\right)$. If $\varphi \leqslant \psi$ in $\mathcal{P}\left(\mathbf{P}_{\mathbf{X}}, \mathbf{D}\right)$, then, for all $x \in X$ we have $\varphi(f(x)) \leqslant \psi(f(x))$ and $\varphi(g(x)) \leqslant \psi(g(x))$ and consequently, as $m$ is order-preserving,

$$
\varphi^{*}(x)=m(\varphi(f(x)), \varphi(g(x))) \leqslant m(\psi(f(x)), \psi(g(x)))=\psi^{*}(x) .
$$

Hence, $\varphi^{*} \leqslant \psi^{*}$ in $\boldsymbol{y}_{h}\left(\mathbf{X}, \mathbf{M}_{h}\right)$. For all $x \in X$, we have

$$
\begin{aligned}
\alpha^{* *}(x) & =\left(\alpha \uparrow_{P_{\mathbf{X}}}\right)^{*}(x) \\
& =m\left(\alpha \uparrow_{P_{\mathbf{X}}}(f(x)), \alpha \uparrow_{P_{\mathbf{X}}}(g(x))\right) \\
& =m(\alpha(f(x)), \alpha(g(x))) \\
& =m(f(\alpha(x)), g(\alpha(x))) \\
& =\alpha(x)
\end{aligned}
$$

by (7) in $M$.

Thus $\alpha^{* *}=\alpha$. For all $u \in P_{\mathbf{X}}$, we have

$$
\begin{array}{rlr}
\varphi^{* *}(u) & =\varphi^{*} \uparrow_{P_{\mathbf{X}}}(u) & \\
& =\varphi^{*}(u) & \\
& =m(\varphi(f(u)), \varphi(g(u))) & \\
& =m(\varphi(u), \varphi(u)) & \text { as } u \in P_{\mathbf{X}}
\end{array}
$$$$
=\varphi(u) \quad \text { by (11) in } M \text {, }
$$

whence $\varphi^{* *}=\varphi$. We have proved that the maps $\Phi: \alpha \mapsto \alpha^{*}$ and $\Psi: \varphi \mapsto \varphi^{*}$ are mutually inverse lattice isomorphisms.

In order to prove that $\varepsilon_{\mathbf{X}}: \mathbf{X} \rightarrow \mathrm{DE}(\mathbf{X})$ is surjective, it remains to show that if $k: \mathrm{E}(\mathbf{X}) \rightarrow \underline{\mathbf{M}}$ is a $\{0,1\}$-lattice homomorphism, then there exists $x \in X$ such that $k=\varepsilon_{\mathbf{X}}(x)$, that is, that $k(\alpha)=\alpha(x)$ for all $\alpha \in \mathbf{y}_{h}\left(\mathbf{X},{\underset{\sim}{\sim}}_{h}\right)$. Define $l:=$ $k \circ \Psi: \mathrm{K}\left(\mathbf{P}_{\mathbf{X}}\right) \rightarrow \underline{\mathbf{M}}$, then $f \circ l \leqslant g \circ l$ in $\operatorname{HK}\left(\mathbf{P}_{\mathbf{X}}\right)$. Since Priestley duality is full, there exist $u, v \in P_{\mathbf{X}}$ such that $u \preccurlyeq v$ and $(f \circ l)(\varphi)=\varphi(u)$ and $(g \circ l)(\varphi)=\varphi(v)$, for all $\varphi \in \mathcal{P}\left(\mathbf{P}_{\mathbf{X}}, \mathbf{D}\right)$. Since $u \preccurlyeq v$, there exists $x \in X$ such that $f(x)=u$ and 
$g(x)=v$. We shall now show that $k(\alpha)=\alpha(x)$ for all $\alpha \in \boldsymbol{y}_{h}\left(\mathbf{X}, \mathbf{M}_{h}\right)$. Let $\alpha \in \boldsymbol{y}_{h}\left(\mathbf{X}, \mathbf{M}_{h}\right)$; then

$$
\begin{aligned}
f\left(l\left(\alpha^{*}\right)\right)=\alpha^{*}(u) \& & g\left(l\left(\alpha^{*}\right)\right)=\alpha^{*}(v) \\
& \Longrightarrow f\left(k(\Psi(\Phi(\alpha)))=\alpha^{*}(u) \& g(k(\Psi(\Phi(\alpha))))=\alpha^{*}(v)\right. \\
& \Longrightarrow f(k(\alpha))=\alpha(u) \& g(k(\alpha))=\alpha(v) \\
& \Longrightarrow f(k(\alpha))=\alpha(f(x)) \& g(k(\alpha))=\alpha(g(x)) \\
& \Longrightarrow f(k(\alpha))=f(\alpha(x)) \& g(k(\alpha))=g(\alpha(x)),
\end{aligned}
$$

whence $k(\alpha)=\alpha(x)$, since $f$ and $g$ separate the points of $M$.

It follows from this proposition that, to complete the proof of Theorem 1, we must construct a structure $\mathbf{X} \in X_{h}$ such that $\mathbf{P}_{\mathbf{X}}$ is not a Priestley space. Let $C$ be the Cantor set, obtained, as usual, from the unit interval $[0,1]$ by the removal of middle thirds, and let $\leqslant$ be the usual order on $C$. Define a new order $\preccurlyeq$ on $C$ by $x \preccurlyeq y$ if and only if $x=y$ or $x$ is covered by $y$ with respect to $\leqslant$. Thus $\langle C ; \preccurlyeq\rangle$ is an ordered set of length one in which $x$ is covered by $y$ if and only if $x$ and $y$ are end points of one of the middle thirds which was removed in the construction of $C$. Let $\mathbf{C}:=\langle C ; \preccurlyeq, \mathcal{T}\rangle$ be the resulting ordered Boolean space. As was first observed by Stralka [15], $\mathbf{C}$ is not totally order-disconnected and therefore is not a Priestley space: if $y>x$ but $y$ does not cover $x$ with respect to $\leqslant$, then $x \npreceq y$ but every clopen downset in $\mathbf{C}$ which contains $y$ also contains $x$. We will construct a structure $\mathbf{X} \in \boldsymbol{X}_{h}:=\mathbb{I S}_{\mathrm{C}} \mathbb{P}^{+}\left(\mathbf{M}_{h}\right)$ such that $\mathbf{P}_{\mathbf{X}}=\mathbf{C}$. Let $\Delta$ be the set of all $d \in[0,1]$ such that $d$ is the midpoint of one of the open intervals $\left(l_{d}, u_{d}\right)$ removed in the construction of $C$. Define $X:=C \cup \Delta$ and endow $X$ with the relative topology, $\mathcal{T}$, from the interval $[0,1]$. Note that $\Delta$ is a discretely topologised, open subset of $X$. Define $f$ and $g$ on $X$ as follows:

$$
f(x)=\left\{\begin{array}{ll}
x & \text { if } x \in C, \\
l_{x} & \text { if } x \in \Delta,
\end{array} \text { and } \quad g(x)= \begin{cases}x & \text { if } x \in C, \\
u_{x} & \text { if } x \in \Delta .\end{cases}\right.
$$

Define the domain of the partial map $h$ by

$$
\operatorname{dom}(h)=\{(c, c) \mid c \in C\} \cup\left\{\left(l_{d}, d\right) \mid d \in \Delta\right\} \cup\left\{\left(d, u_{d}\right) \mid d \in \Delta\right\},
$$

and define $h: \operatorname{dom}(h) \rightarrow X$ by $h(c, c)=c, h\left(l_{d}, d\right)=d$ and $h\left(d, u_{d}\right)=d$. Define $\mathbf{X}:=\langle X ; f, g, h, \mathcal{T}\rangle$ and note that, for each $d \in \Delta$, the subset $Z_{d}:=\left\{l_{d}, d, u_{d}\right\}$ forms a closed substructure $\mathbf{Z}_{d}$ of $\mathbf{X}$ which is isomorphic to $\mathbf{M}_{h}$.

Lemma 3.3. The structure $\mathbf{X}:=\langle X ; f, g, h, \mathcal{T}\rangle$ defined above belongs to $\boldsymbol{X}_{h}$, but the corresponding compact ordered space $\mathbf{P}_{\mathbf{X}}$ is not a Priestley space.

Proof. Since it is clear that $\mathbf{P}_{\mathbf{X}}=\mathbf{C}$, it remains to verify that $\mathbf{X}$ is isomorphic to a closed substructure of $\mathbf{M}_{h}^{S}$ for some set $S$. 
For each $d \in \Delta$, define $\alpha_{d}: X \rightarrow M$ by

$$
\alpha_{d}(x)= \begin{cases}1 & \text { if } x \geqslant u_{d} \\ a & \text { if } x=d \\ 0 & \text { if } x \leqslant l_{d}\end{cases}
$$

Now let $d \in \Delta$. We want to show that $\alpha_{d} \in \boldsymbol{X}_{h}\left(\mathbf{X}, \mathbf{M}_{h}\right)$. Clearly $\alpha_{d}$ is continuous and preserves both $f$ and $g$. To check that $\alpha_{d}$ preserves $h$, let $(x, y) \in \operatorname{dom}(h)$. If $(x, y)=(c, c)$, for some $c \in C$, then $\left(\alpha_{d}(x), \alpha_{d}(y)\right) \in\{(0,0),(1,1)\} \subseteq \operatorname{dom}(h)$, and $\alpha_{d}(h(x, y))=\alpha_{d}(x)=h\left(\alpha_{d}(x), \alpha_{d}(y)\right)$. We can now assume that $x, y \in Z_{d^{\prime}}$ for some $d^{\prime} \in \Delta$. The map $\alpha_{d}\left\lceil_{Z_{d^{\prime}}}\right.$ is either constant onto $\{0\}$, constant onto $\{1\}$ or the isomorphism of $\mathbf{Z}_{d}$ onto $\mathbf{M}_{h}$. So $\alpha_{d}$ preserves $h$.

For all $x<y$ in $X$, there exists $d \in \Delta$ with $x \leqslant d \leqslant y$ and consequently $\alpha_{d}(x) \neq \alpha_{d}(y)$. To prove that $\mathbf{X}$ is isomorphic to a closed substructure of a power of $\mathbf{M}_{h}$ it only remains to prove that if $(x, y) \in X^{2} \backslash \operatorname{dom}(h)$, then there exists a continuous morphism $\alpha: \mathbf{X} \rightarrow \mathbb{M}_{h}$ with $(\alpha(x), \alpha(y)) \in M^{2} \backslash \operatorname{dom}(h)$ : see the Separation Theorem 1.4.4 in [2]. Let $(x, y) \in X^{2} \backslash \operatorname{dom}(h)$. First assume that $x<y$. Since $(x, y) \notin \operatorname{dom}(h)$, there is some $d \in \Delta$ with $x<d<y$. It follows that $\left(\alpha_{d}(x), \alpha_{d}(y)\right)=(0,1) \notin \operatorname{dom}(h)$. Now assume that $x>y$. Choose $d \in \Delta$ with $x \geqslant d \geqslant y$. Then $\alpha_{d}(x)>\alpha_{d}(y)$, whence $\left(\alpha_{d}(x), \alpha_{d}(y)\right) \notin \operatorname{dom}(h)$. Finally, assume that $x=y$. Then $x \in \Delta$ and consequently $\left(\alpha_{x}(x), \alpha_{x}(y)\right)=(a, a) \notin \operatorname{dom}(h)$. Thus, $\mathbf{X} \in \mathbb{\mathbb { S } _ { \mathrm { c } }} \mathbb{P}^{+}\left(\mathbb{M}_{h}\right)$, as claimed.

\section{Structural entailment: a sneak preview}

Entailment and hom-entailment play important roles in the study of dualities and strong dualities, respectively: see Chapters 8 and 9 in Clark and Davey [2]. Before we prove Theorem 2, we need to introduce a form of entailment that appears to be important in the study of full dualities. A more detailed discussion will appear in [10].

Let $\underset{\sim}{\mathbf{M}}=\langle M ; G, H, R, \mathcal{T}\rangle$ be an alter ego of a finite algebra $\underline{\mathbf{M}}$ and let $s$ be an algebraic relation on $\underline{\mathbf{M}}$. Given a closed substructure $\mathbf{X}$ of a non-zero power of $\underset{\sim}{\mathbf{M}}$, we say that $G \cup H \cup R$, or simply $\underset{\sim}{\mathbf{M}}$, entails $s$ on $\mathbf{X}$ if each morphism $\alpha: \mathbf{X} \rightarrow \underset{\sim}{\mathbf{M}}$ preserves $s$. We shall say that $\underset{\sim}{\mathbf{M}}$ structurally entails $s$ if $\underset{\sim}{\mathbf{M}}$ entails $s$ on $\mathbf{X}$, for every closed substructure $\mathbf{X}$ of a non-zero power of $\underset{\sim}{\mathbf{M}}$. Recall that $\underset{\sim}{\mathbf{M}}$ entails $s$ precisely when it entails $s$ on every structure of the form $D(\mathbf{A})$, for $\mathbf{A} \in \mathbb{I S P}(\underline{\mathbf{M}})$. Hence it is trivial that if $\underset{\sim}{\mathbf{M}}$ structurally entails $s$, then $\underset{\sim}{\mathbf{M}}$ entails $s$. The converse statement is not true; for example, $\underset{\sim}{\mathbf{M}}$, as defined in Section 1, entails the order relation $\leqslant$, but for the substructure $\{0,1\}$ of $\underset{\sim}{\mathbf{M}}$, the $\underset{\sim}{\mathbf{M}}$-morphism $\{0,1\} \rightarrow\{0,1\}$ given by Boolean complementation does not preserve $\leqslant$. 
Now assume that $s$ is $n$-ary and let $\mathbf{s}$ be the corresponding subalgebra of $\underline{\mathbf{M}}^{n}$. For $i=1, \ldots, n$, let $\rho_{i}=\pi_{i} \uparrow_{\mathbf{s}}: \mathbf{s} \rightarrow \underline{\mathbf{M}}$ be the natural projection. We wish to describe the substructure of $\underset{\sim}{\mathbf{M}}$ generated by the set $\left\{\rho_{1}, \ldots, \rho_{n}\right\}$. Since $\mathrm{D}(\mathbf{s})$ is a substructure of $\underset{\sim}{\mathbf{M}}{ }^{s}$ containing $\left\{\rho_{1}, \ldots, \rho_{n}\right\}$, it suffices to describe the substructure $\operatorname{sg}_{\mathrm{D}(\mathbf{s})}\left(\left\{\rho_{1}, \ldots, \rho_{n}\right\}\right)$ of $\mathrm{D}(\mathbf{s})$.

The enriched partial clone generated by $G \cup H$ is denoted by $[G \cup H]$ and is referred to as the enriched partial clone of $\underset{\sim}{\mathbf{M}}$. The set of all $n$-ary maps in $[G \cup H]$ is denoted by $[G \cup H]_{n}$. (See pages 21, 64 and 278-283 of Clark and Davey [2] for a discussion of the enriched partial clone of $\underset{\sim}{\mathcal{M}}$.)

Lemma 4.1. Let $\underline{\mathbf{M}}$ be a finite algebra, let $\underset{\sim}{\mathbf{M}}$ be an alter ego of $\underline{\mathbf{M}}$ and let $s$ be an $n$-ary algebraic relation on $\underline{\mathbf{M}}$. The substructure of $\mathrm{D}(\mathbf{s})$ generated by $\left\{\rho_{1}, \ldots, \rho_{n}\right\}$ consists of all homomorphisms from $\mathbf{s}$ to $\underline{\mathbf{M}}$ that have an extension in the enriched partial clone of $\underset{\sim}{\mathbf{M}}$. Thus,

$$
\operatorname{sg}_{\mathrm{D}(\mathbf{s})}\left(\left\{\rho_{1}, \ldots, \rho_{n}\right\}\right)=\left\{t \uparrow_{s}: s \rightarrow M \mid t \in[G \cup H]_{n} \& s \subseteq \operatorname{dom}(t)\right\} .
$$

Proof. It is easy to verify that $\operatorname{sg}_{\mathrm{D}(\mathbf{s})}\left(\left\{\rho_{1}, \ldots, \rho_{n}\right\}\right)$ equals

$$
\left\{t^{\mathrm{D}(\mathbf{s})}\left(\rho_{1}, \ldots, \rho_{n}\right) \mid t \in[G \cup H]_{n} \quad \&\left(\rho_{1}, \ldots, \rho_{n}\right) \in \operatorname{dom}\left(t^{\mathrm{D}(\mathbf{s})}\right)\right\} .
$$

Thus, we must check that, for all $t \in[G \cup H]_{n}$, we have $s \subseteq \operatorname{dom}(t)$ if and only if $\left(\rho_{1}, \ldots, \rho_{n}\right) \in \operatorname{dom}\left(t^{\mathrm{D}(\mathbf{s})}\right)$. We must also check that, for all $t \in[G \cup H]_{n}$, we have $t^{\mathrm{D}(\mathbf{s})}\left(\rho_{1}, \ldots, \rho_{n}\right)=t \uparrow_{s}$. These simple calculations, which are left to the reader, use only the fact that $\left(\rho_{1}(c), \ldots, \rho_{n}(c)\right)=c$, for all $c \in s$.

We leave the proof of the following result as an easy exercise for the reader.

Lemma 4.2. Let $\underset{\sim}{\mathbf{M}}$ be an alter ego of a finite algebra $\underline{\mathbf{M}}$ and let $s$ be an $n$-ary algebraic relation on $\underline{\mathbf{M}}$. If $s$ is definable in the language of $\underset{\sim}{\mathbf{M}}$ via a conjunct of atomic formula in $n$ free variables, then $\underset{\sim}{\mathbf{M}}$ structurally entails $s$.

Proposition 4.3. Let $\underset{\sim}{\mathbf{M}}$ be an alter ego of a finite algebra $\underline{\mathbf{M}}$ and assume that $\underset{\sim}{\mathbf{M}}$ fully dualises $\underline{\mathbf{M}}$ at the finite level.

(a) Let $n \in \mathbb{N}$ and let $s$ be an $n$-ary algebraic relation on $\underline{\mathbf{M}}$. If $\underset{\sim}{\mathbf{M}}$ structurally entails $s$, then every $n$-ary algebraic partial operation on $\underline{\mathbf{M}}$ with domain $s$ has an extension in the enriched partial clone of $\underset{\sim}{\mathbf{M}}$.

(b) Every total algebraic operation on $\underline{\mathbf{M}}$ is in the enriched partial clone of $\underset{\sim}{\mathbf{M}}$.

Proof. Fix $n \in \mathbb{N}$ and assume that $s$ is an $n$-ary algebraic relation on $\underline{\mathbf{M}}$ that is structurally entailed by $\underset{\sim}{\mathbf{M}}$. Let $\mathbf{X}:=\mathbf{s g}_{\mathrm{D}(\mathbf{s})}\left(\left\{\rho_{1}, \ldots, \rho_{n}\right\}\right)$. By Lemma 4.1 , in order to prove (a), it suffices to prove that $\mathbf{X}=\mathrm{D}(\mathbf{s})$.

Since $\left(\rho_{1}(c), \ldots, \rho_{n}(c)\right)=c$, for all $c \in s$, we have $\left(\rho_{1}, \ldots, \rho_{n}\right) \in s^{\mathrm{D}(\mathbf{s})}$. Hence, $\left(\rho_{1}, \ldots, \rho_{n}\right) \in s^{\mathbf{X}}$, as $\mathbf{X}$ is a substructure of $\mathrm{D}(\mathbf{s})$. Let $\alpha$ be an element of $\mathrm{E}(\mathbf{X})$, that is, assume $\alpha: \mathbf{X} \rightarrow \underset{\sim}{\mathbf{M}}$ is a morphism. As $\underset{\sim}{\mathbf{M}}$ structurally entails $s$, it follows 
that $\alpha$ preserves $s$. Since $\left(\rho_{1}, \ldots, \rho_{n}\right) \in s^{\mathbf{X}}$, we have $\left(\alpha\left(\rho_{1}\right), \ldots, \alpha\left(\rho_{n}\right)\right) \in s$. Thus, $u: \mathrm{E}(\mathbf{X}) \rightarrow \mathbf{s}$, given by $u(\alpha):=\left(\alpha\left(\rho_{1}\right), \ldots, \alpha\left(\rho_{n}\right)\right)$, for all $\alpha$ in the algebra $\mathrm{E}(\mathbf{X})$, is a well-defined homomorphism. Since $\mathbf{X}$ is generated by $\left\{\rho_{1}, \ldots, \rho_{n}\right\}$, the map $u$ is one-to-one. To see that $u$ is surjective, let $c \in s$ and define $\alpha:=e_{\mathbf{s}}(c)\left\lceil_{\mathbf{X}}: \mathbf{X} \rightarrow \underset{\sim}{\mathbf{M}}\right.$, where $e_{\mathbf{s}}: \mathbf{s} \rightarrow \mathrm{ED}(\mathbf{s})$ is the natural isomorphism. Then $\alpha$ belongs to the algebra $\mathrm{E}(\mathbf{X})$ and

$$
u(\alpha)=\left(\alpha\left(\rho_{1}\right), \ldots, \alpha\left(\rho_{n}\right)\right)=\left(e_{\mathbf{s}}(c)\left(\rho_{1}\right), \ldots, e_{\mathbf{s}}(c)\left(\rho_{n}\right)\right)=\left(\rho_{1}(c), \ldots, \rho_{n}(c)\right)=c .
$$

Consequently, $u: \mathrm{E}(\mathbf{X}) \rightarrow \mathbf{s}$ is an isomorphism. Because $\underset{\sim}{\mathbf{M}}$ fully dualises $\underline{\mathbf{M}}$ at the finite level, we get $\mathbf{X} \cong \mathrm{DE}(\mathbf{X}) \cong \mathrm{D}(\mathbf{s})$. But $\mathbf{X} \leqslant \mathrm{D}(\mathbf{s})$ and both are finite, so by cardinality considerations we have $\mathbf{X}=\mathrm{D}(\mathbf{s})$. This proves (a).

Since $M^{n}$ is clearly definable via a conjunct of atomic formulæ, it follows immediately from (a) and the previous lemma that (b) holds.

\section{The proof of Theorem 2}

Throughout this section, $\underset{\sim}{\mathbf{M}}$ is a fixed alter ego which fully dualises the threeelement chain $\underline{\mathbf{M}}=\langle\{0, a, 1\} ; \vee, \wedge, 0,1\rangle$. By Proposition 4.3, the endomorphisms $f$ and $g$ are in the enriched partial clone of $\mathbf{\sim}$ and so can be considered, with no loss of generality, to be in the type of $\underset{\sim}{\mathbf{M}}$. Since $\underset{\sim}{\mathbf{M}_{m}}=\langle M ; f, g, m, \mathcal{T}\rangle$ strongly dualises $\underline{\mathbf{M}}$, to prove that $\underset{\sim}{\mathbf{M}}$ strongly dualises $\underline{\mathbf{M}}$ it now suffices to prove that $m$ has an extension in the enriched partial clone of $\underset{\sim}{\mathbf{M}}$. Note that, since we do not know the language of $\underset{\sim}{\mathbb{M}}$ precisely, we do not know that $\operatorname{dom}(m)=\leqslant\{0,1\}$ is appropriately definable in the language of $\underset{\sim}{\mathbf{M}}$ and, consequently, we cannot apply Proposition 4.3 to the partial operation $m$. We must work harder, and not at the finite level.

Let $\mathbf{I}$ be the bounded distributive lattice with universe $I=[0,1] \cap \mathbb{Q}$ and with its order inherited from $\mathbb{R}$. We shall describe some elements of the underlying set $\mathcal{D}(\mathbf{I}, \underline{\mathbf{M}})$ of the dual $\mathrm{D}(\mathbf{I})$. For each real number $r \in(0,1)$ define $x_{r} \in \mathcal{D}(\mathbf{I}, \underline{\mathbf{M}})$ by

$$
x_{r}(q)= \begin{cases}0 & \text { if } q<r \\ a & \text { if } q=r \\ 1 & \text { if } q>r\end{cases}
$$

Note that the range of $x_{r}$ is $\{0, a, 1\}$ if $r \in \mathbb{Q}$ and is $\{0,1\}$ otherwise. For $r \in[0,1] \cap \mathbb{Q}$ and $k \in\{0,1\}$, define $y_{r}^{k} \in \mathcal{D}(\mathbf{I}, \underline{\mathbf{M}})$ by

$$
y_{r}^{k}(q)= \begin{cases}0 & \text { if } q<r \\ k & \text { if } q=r \\ 1 & \text { if } q>r\end{cases}
$$


We shall focus our attention on the subset

$$
Y:=\left\{x_{r} \mid r \in(0,1)\right\} \cup\left\{y_{r}^{0} \mid r \in[0,1) \cap \mathbb{Q}\right\} \cup\left\{y_{r}^{1} \mid r \in(0,1] \cap \mathbb{Q}\right\}
$$

of $\mathcal{D}(\mathbf{I}, \underline{\mathbf{M}})$ and on the closed substructure $\mathbf{X}$ of $\mathrm{D}(\mathbf{I})$ that it generates.

\section{Lemma 5.1.}

(a) For each $z \in \mathcal{D}(\mathbf{I}, \underline{\mathbf{M}}) \backslash Y$ there exist rational numbers $r, s$ with $0<r<s<1$ such that $[r, s] \cap \mathbb{Q} \subseteq z^{-1}(a)$.

(b) The set $Y$ is a topologically closed proper subset of $\mathcal{D}(\mathbf{I}, \underline{\mathbf{M}})$.

Proof. The first claim is clearly true as is the fact that $Y$ is a proper subset of $\mathcal{D}(\mathbf{I}, \underline{\mathbf{M}})$. We now prove that $Y$ is topologically closed in $\mathcal{D}(\mathbf{I}, \underline{\mathbf{M}})$. The subbasic clopen sets in $\mathcal{D}(\mathbf{I}, \underline{\mathbf{M}})$ are of all the form

$$
U_{r, m}:=\{z \in \mathcal{D}(\mathbf{I}, \underline{\mathbf{M}}) \mid z(r)=m\},
$$

for $r \in I$ and $m \in M=\{0, a, 1\}$. Let $z \in \mathrm{D}(\mathbf{I}) \backslash Y$. By (a), there exist $r, s \in z^{-1}(a)$ with $r \neq s$. Thus, $U_{r, a} \cap U_{s, a}$ is a clopen subset of $\mathcal{D}(\mathbf{I}, \underline{\mathbf{M}})$ that contains $z$ and is disjoint from $Y$. Hence, $Y$ is topologically closed in $\mathcal{D}(\mathbf{I}, \underline{\mathbf{M}})$.

Recall that we are assuming that $\underset{\sim}{\mathbf{M}}$ fully dualises $\underline{\mathbf{M}}$ and that we need to show that the partial binary operation $m$ can be extended to a member of the enriched partial clone of $\underset{\sim}{\mathbf{M}}$. Define $\mathbf{X}$ to be the topologically closed substructure of $\mathrm{D}(\mathbf{I})$ generated by $Y$. The main step of the argument is to show that $X \neq Y$ by showing $\mathbf{X}=\mathrm{D}(\mathbf{I})$. (This step uses full duality.) This will suffice, as we now show.

Lemma 5.2. If $X \neq Y$, then $m$ can be extended to a member of the enriched partial clone of $\underset{\sim}{\mathbf{M}}$.

Proof. Assume that $X \neq Y$. Since $Y$ is a topologically closed subset of $\mathcal{D}(\mathbf{I}, \underline{\mathbf{M}})$, the set $Y$ must fail to be closed under some partial operation $p$ in the type of $\underset{\sim}{\mathbf{M}}$. Let the arity of $p$ be $n$, and choose $y_{1}, \ldots, y_{n} \in Y$ so that $p\left(y_{1}, \ldots, y_{n}\right)$ is defined and $p\left(y_{1}, \ldots, y_{n}\right) \in X \backslash Y$. Hence, by Lemma 5.1(a), there exist $r, s \in I$ with $r<s$ such that $[r, s] \cap \mathbb{Q} \subseteq p\left(y_{1}, \ldots, y_{n}\right)^{-1}(a)$.

By the definition of $Y$, we have $\left|y_{i}^{-1}(a)\right| \leqslant 1$, for $i=1, \ldots, n$. Since the set $y_{1}^{-1}(a) \cup \cdots \cup y_{n}^{-1}(a)$ is finite, there exists $t \in([r, s] \cap \mathbb{Q}) \backslash\left(y_{1}^{-1}(a) \cup \cdots \cup y_{n}^{-1}(a)\right)$. Hence,

$$
p\left(y_{1}, \ldots, y_{n}\right)(t)=a \text { and } y_{1}(t), \ldots, y_{n}(t) \in\{0,1\}
$$

For each $i=1, \ldots, n$, define $c_{i}:=y_{i}(t) \in\{0,1\}$. Then $\left(c_{1}, \ldots, c_{n}\right) \in \operatorname{dom}(p)$ with $p\left(c_{1}, \ldots, c_{n}\right)=a$. Since $p(0, \ldots, 0)=0$ and $p(1, \ldots, 1)=1$, there must exist $i$ and $j$ such that $c_{i}=0$ and $c_{j}=1$. By rearranging the variables of $p$ if necessary, we 
conclude that

$$
\begin{aligned}
& p(0, \ldots, 0,0, \ldots, 0)=0, \\
& p(0, \ldots, 0,1, \ldots, 1)=a, \\
& p(1, \ldots, 1,1, \ldots, 1)=1 .
\end{aligned}
$$

Collapsing variables yields an extension of $m$ in the enriched partial clone of $\underset{\sim}{\mathbf{M}}$, as required.

We now work towards showing that $\mathbf{X}=\mathrm{D}(\mathbf{I})$. Define the unary relation $D$ and the ternary relation $s$ by

$$
\begin{aligned}
D & =\operatorname{fix}(f)=\{0,1\}, \\
s & =\operatorname{graph}(m)=\{(0,0,0),(0,1, a),(1,1,1)\} .
\end{aligned}
$$

Lemma 5.3. The relations $D$ and $s$ are definable via conjuncts of equations using $f$ and $g$, and hence $\underset{\sim}{\mathbf{M}}$ structurally entails $D$ and $s$.

Proof. Clearly, $s$ is defined by " $f\left(x_{3}\right)=x_{1} \quad \& \quad g\left(x_{3}\right)=x_{2}$ " and $D$ is defined by $" f\left(x_{1}\right)=x_{1} "$. Now apply Lemma 4.2.

As $\mathbf{X}$ is a substructure of $\mathrm{D}(\mathbf{I})$, the maps $e_{\mathbf{I}}(q)\left\lceil_{X}: X \rightarrow M\right.$ and $e_{\mathbf{I}}(q)\left\lceil_{Y}: Y \rightarrow M\right.$ are well defined, for all $q \in I$, where $e_{\mathbf{I}}: \mathbf{I} \rightarrow \operatorname{DE}(\mathbf{I})$ is the natural isomorphism.

Lemma 5.4. If $\alpha: Y \rightarrow M$ is continuous and preserves $D$ and $s$, interpreted coordinatewise, then there exists $q \in I$ such that $\alpha=e_{\mathbf{I}}(q) \Gamma_{Y}$.

Proof. Let $\alpha: Y \rightarrow M$ satisfy the hypotheses of the lemma. Define $\Phi:[0,1] \rightarrow M$ by

$$
\Phi(r)= \begin{cases}\alpha\left(x_{r}\right) & \text { if } 0<r<1 \\ \alpha\left(y_{0}^{0}\right) & \text { if } r=0 \\ \alpha\left(y_{1}^{1}\right) & \text { if } r=1\end{cases}
$$

We shall study $\Phi$ quite closely. Firstly, note that for all $r \in(0,1) \backslash \mathbb{Q}$, the map $x_{r}$ is coordinatewise in $D$ and therefore $\alpha\left(x_{r}\right) \in D$. So

$$
\Phi(r) \in\{0,1\} \text {, for } r \in(0,1) \backslash \mathbb{Q} .
$$

Similarly,

$$
\Phi(0), \Phi(1) \in\{0,1\}
$$

Secondly, note that if $r \in(0,1) \cap \mathbb{Q}$, then $\left(y_{r}^{0}, y_{r}^{1}, x_{r}\right)$ is coordinatewise in $s$, so we get

$$
\left(\alpha\left(y_{r}^{0}\right), \alpha\left(y_{r}^{1}\right), \alpha\left(x_{r}\right)\right) \in s
$$


This means that, for $r \in(0,1) \cap \mathbb{Q}$, we have

$$
\begin{array}{lll}
\Phi(r)=0 & \text { implies } & \alpha\left(y_{r}^{0}\right)=\alpha\left(y_{r}^{1}\right)=0, \\
\Phi(r)=a & \text { implies } & \alpha\left(y_{r}^{0}\right)=0 \text { and } \alpha\left(y_{r}^{1}\right)=1, \\
\Phi(r)=1 & \text { implies } & \alpha\left(y_{r}^{0}\right)=\alpha\left(y_{r}^{1}\right)=1 .
\end{array}
$$

It follows from (5.3)-(5.5) that $\Phi$ determines $\alpha$.

Recall that we want to show that $\alpha=e_{\mathbf{I}}(q) \uparrow_{Y}$ for some $q \in I$. By (5.3)-(5.5), this is equivalent to showing that one of the following conditions holds:

(i) $\Phi$ is the constant 0 function (giving $\left.\alpha=e_{\mathbf{I}}(0) \uparrow_{Y}\right)$;

(ii) $\Phi$ is the constant 1 function (giving $\left.\alpha=e_{\mathbf{I}}(1) \uparrow_{Y}\right)$;

(iii) there exists $q \in(0,1) \cap \mathbb{Q}$ such that

$$
\Phi(r)= \begin{cases}1 & \text { if } r<q \\ a & \text { if } r=q \\ 0 & \text { if } r>q\end{cases}
$$

(giving $\left.\alpha=e_{\mathbf{I}}(q) \uparrow_{Y}\right)$.

In order to use the continuity of $\alpha$, we need the following readily established topological facts about $Y$.

Claim. Let $V$ be a (relatively) open subset of $Y$ and let $r \in[0,1]$.

(a) Assume that $r \in[0,1) \cap \mathbb{Q}$ and $y_{r}^{0} \in V$. There exists $\delta>0$ such that, for all $s \in(r, r+\delta)$ and all $t \in(r, r+\delta) \cap \mathbb{Q}$, we have $x_{s} \in V$ and $y_{t}^{0}, y_{t}^{1} \in V$.

(b) Assume that $r \in(0,1] \cap \mathbb{Q}$ and $y_{r}^{1} \in V$. There exists $\delta>0$ such that, for all $s \in(r-\delta, r)$ and all $t \in(r-\delta, r) \cap \mathbb{Q}$, we have $x_{s} \in V$ and $y_{t}^{0}, y_{t}^{1} \in V$.

(c) Assume that $r \in(0,1) \backslash \mathbb{Q}$ and $x_{r} \in V$. There exists $\delta>0$ such that, for all $s \in(r-\delta, r+\delta)$ and all $t \in(r-\delta, r+\delta) \cap \mathbb{Q}$, we have $x_{s} \in V$ and $y_{t}^{0}, y_{t}^{1} \in V$.

Combining this claim with (5.1)-(5.5) and the continuity of $\alpha$, we can deduce the following facts (which we also leave to the reader).

(d) $\Phi^{-1}(0)$ and $\Phi^{-1}(1)$ are open subsets of $[0,1]$.

(e) (i) $\Phi^{-1}(a) \subseteq(0,1) \cap \mathbb{Q}$,

(ii) if $r \in \Phi^{-1}(a)$, then there exists $\delta>0$ such that $(r-\delta, r) \subseteq \Phi^{-1}(1)$ and $(r, r+\delta) \subseteq \Phi^{-1}(0)$.

Now assume that $\Phi$ is neither constantly 0 nor constantly 1 . If $\Phi^{-1}(a)=\varnothing$, then, by (d), $\left\{\Phi^{-1}(0), \Phi^{-1}(1)\right\}$ is a non-trivial partition of $[0,1]$ into two open subsets, which is impossible as $[0,1]$ is connected. Thus, $\Phi^{-1}(a) \neq \varnothing$. Choose $q \in \Phi^{-1}(a) \subseteq(0,1) \cap \mathbb{Q}$. Define $q^{\top}:=\bigvee\left\{r \in(q, 1] \mid(q, r] \subseteq \Phi^{-1}(0)\right\}$. By (e), this join is over a non-empty set. Since $\Phi^{-1}(1)$ is open, we must have $\Phi\left(q^{\top}\right) \neq 1$. By (e), we must have $\Phi\left(q^{\top}\right) \neq a$. So $\Phi\left(q^{\top}\right)=0$. Since $\Phi^{-1}(0)$ is open, it follows 
that $q^{\top}=1$. Hence, $(q, 1] \subseteq \Phi^{-1}(0)$. Similarly, $[0, q) \subseteq \Phi^{-1}(1)$. Thus, we have $\Phi^{-1}(a)=\{q\}, \Phi^{-1}(1)=[0, q)$ and $\Phi^{-1}(0)=(q, 1]$, which finishes the proof of the lemma.

Recall that the underlying set of the distributive lattice $\mathrm{E}(\mathbf{X})$ is the set $\boldsymbol{X}(\mathbf{X}, \underset{\sim}{\mathbf{M}})$ of all continuous morphisms from $\mathbf{X}$ to $\underset{\sim}{\mathbf{M}}$.

Corollary 5.5. $\boldsymbol{X}(\mathbf{X}, \underset{\sim}{\mathbf{M}})=\left\{\left.e_{\mathbf{I}}(r)\right|_{X} \mid r \in I\right\}$.

Proof. Clearly $\left\{\left.e_{\mathbf{I}}(r)\right|_{X} \mid r \in I\right\} \subseteq \mathcal{X}(\mathbf{X}, \underset{\sim}{\mathbf{M}})$. Let $\alpha \in \boldsymbol{X}(\mathbf{X}, \underset{\sim}{\mathbf{M}})$. Then $\alpha$ is continuous and preserves $D$ and $s$ (by Lemma 5.3). Hence $\left.\alpha\right|_{Y}$ has the same properties. By Lemma 5.4, there exists $r \in I$ such that $\left.\alpha\right|_{Y}=\left.e_{\mathbf{I}}(r)\right|_{Y}$. It follows that $\alpha=\left.e_{\mathbf{I}}(r)\right|_{\mathbf{X}}$, since the equalizer, $\mathbf{Z}$, of $\alpha: \mathbf{X} \rightarrow \underset{\sim}{\mathbf{M}}$ and $e_{\mathbf{I}}(r) \Gamma_{\mathbf{X}}: \mathbf{X} \rightarrow \underset{\sim}{\mathbf{M}}$ is a closed substructure of $\mathbf{X}$ that contains $Y$, forcing $\mathbf{Z}=\mathbf{X}$.

Lemma 5.6. $\mathrm{X}=\mathrm{D}(\mathrm{I})$.

Proof. Define $\rho: I \rightarrow \boldsymbol{X}(\mathbf{X}, \underset{\sim}{\mathbf{M}})$ by $\rho(r)=\left.e_{\mathbf{I}}(r)\right|_{\mathbf{X}}$, for all $r \in I$. By Corollary 5.5, the map $\rho$ is surjective. It is also injective, since if $r, s \in I$ with $r<s$, then, for example, $\left.e_{\mathbf{I}}(r)\right|_{\mathbf{X}}\left(y_{s}^{1}\right)=0 \neq 1=\left.e_{\mathbf{I}}(s)\right|_{\mathbf{X}}\left(y_{s}^{1}\right)$. Further, $\rho$ is a bounded-lattice homomorphism since $X \subseteq \mathcal{D}(\mathbf{I}, \underline{\mathbf{M}})$. Thus $\rho$ is an isomorphism from $\mathbf{I}$ onto $\mathrm{E}(\mathbf{X})$.

Let $y \in \mathcal{D}(\mathbf{I}, \underline{\mathbf{M}})$. (We must show that $y \in X$.) Let $\varphi:=y \circ \rho^{-1}: \mathrm{E}(\mathbf{X}) \rightarrow \underline{\mathbf{M}}$. Since $\underset{\sim}{\mathbf{M}}$ fully dualises $\underline{\mathbf{M}}$, there must exist $x \in X$ such that $\varphi=\varepsilon_{\mathbf{X}}(x)$. Thus $y=\varepsilon_{\mathbf{X}}(x) \circ \rho$, which gives $y=x$, so $y \in X$ as required.

We have proved Theorem 2 .

\section{The characterizations of the dual categories}

In this final section, $\underset{\sim}{\mathbf{M}}, \underset{\sim}{\underset{\sim}{\mathbf{M}}}$ and $\underset{\sim}{\mathbf{M}} \boldsymbol{m}$ refer to the alter egos introduced in Section 1. We shall characterize the members of the topological quasi-varieties $\boldsymbol{X}=\mathbb{I} \mathbb{S}_{c} \mathbb{P}^{+}(\underset{\sim}{\mathbf{M}})$, $x_{h}=\mathbb{I S}_{\mathrm{c}} \mathbb{P}^{+}\left({\underset{\sim}{\sim}}_{h}\right)$ and $\boldsymbol{X}_{m}=\mathbb{I S}_{\mathrm{c}} \mathbb{P}^{+}\left(\underset{\sim}{\mathbf{M}_{m}}\right)$ relative to the corresponding topological quasi-equational classes $\boldsymbol{y}=\operatorname{Mod}_{\mathcal{T}}(\Sigma), \boldsymbol{y}_{h}=\operatorname{Mod}_{\mathcal{T}}\left(\Sigma_{h}\right)$ and $\boldsymbol{y}_{m}=\operatorname{Mod}_{\mathcal{T}}\left(\Sigma_{m}\right)$, as introduced in Section 2. In each case, the given quasi-equations are insufficient to characterise the topological quasi-variety and a further separation condition on the "ordered" space $\mathbf{P}_{\mathbf{X}}$ is required.

Recall that, for all $\mathbf{X} \in \mathbf{y}$, we define $\mathbf{P}_{\mathbf{X}}:=\left\langle P_{\mathbf{X}} ; \preccurlyeq, \mathcal{T}\right\rangle$, where $P_{\mathbf{X}}:=f i x(f) \subseteq X$ and $\preccurlyeq$ is defined on $P_{\mathbf{X}}$ by

$$
u \preccurlyeq v \Longleftrightarrow(\exists x \in X) f(x)=u \quad \& g(x)=v .
$$

By Lemma 3.1, the relation $\preccurlyeq$ is reflexive and anti-symmetric, and is transitive, and therefore an order relation, provided $\mathbf{X} \in \mathbf{y}_{h}$. We say that a subset $U \subseteq P_{\mathbf{X}}$ is a downset of $\mathbf{P}_{\mathbf{X}}$ if $u \preccurlyeq v$ and $v \in U$ imply $u \in U$, for all $u, v \in P_{\mathbf{X}}$. A pair $x, y$ of 
distinct points in $P_{\mathbf{X}}$ is separated by a clopen downset of $\mathbf{P}_{\mathbf{X}}$ provided there exists a clopen downset $U$ of $\mathbf{P}_{\mathbf{X}}$ containing exactly one of $x$ and $y$.

We start by characterizing the members of the topological quasi-variety $\boldsymbol{X}$. This result was obtained while the first and second authors were working with David Clark on part of [3].

Lemma 6.1. Let $\mathbf{X} \in \mathbf{y}$ and assume that each pair of distinct points of $P_{\mathbf{X}}$ is separated by a clopen downset of $\mathbf{P}_{\mathbf{X}}$. If $x \neq y$ in $X$, then there is a morphism $\alpha \in \mathbf{y}(\mathbf{X}, \underset{\sim}{\mathbf{M}})$ such that $\alpha(x) \neq \alpha(y)$.

Proof. We first assume that $x, y \in P_{\mathbf{X}}$. By hypothesis we may assume that there is a clopen downset $U$ of $\mathbf{P}_{\mathbf{X}}$ containing $y$ and not $x$. Let $V:=P_{\mathbf{X}} \backslash U$. Then there are open sets $U_{1}, V_{1} \subseteq X$ with $U=P_{\mathbf{X}} \cap U_{1}, V=P_{\mathbf{X}} \cap V_{1}$. We partition $\mathbf{X}$ into four open, and therefore clopen, sets:

$$
X=W_{0} \dot{\cup} W_{a} \dot{\cup} W_{b} \dot{\cup} W_{1},
$$

where

$$
\begin{aligned}
& W_{0}:=f^{-1}\left(U_{1}\right) \cap g^{-1}\left(U_{1}\right)=f^{-1}(U) \cap g^{-1}(U) \supseteq U, \\
& W_{a}:=f^{-1}\left(U_{1}\right) \cap g^{-1}\left(V_{1}\right)=f^{-1}(U) \cap g^{-1}(V), \\
& W_{b}:=f^{-1}\left(V_{1}\right) \cap g^{-1}\left(U_{1}\right)=f^{-1}(V) \cap g^{-1}(U), \\
& W_{1}:=f^{-1}\left(V_{1}\right) \cap g^{-1}\left(V_{1}\right)=f^{-1}(V) \cap g^{-1}(V) \supseteq V .
\end{aligned}
$$

(This uses quasi-equations (1) and (2).) However, $W_{b}=\varnothing$; indeed, if $f(z) \in V$ and $g(z) \in U$, then, as $U$ is a downset and $f(z) \preccurlyeq g(z)$, we obtain $f(z) \in U \cap V$, a contradiction. Hence $X=W_{0} \dot{\cup} W_{a} \dot{\cup} W_{1}$. We define a map $\alpha: X \rightarrow M$ by

$$
\alpha(x)= \begin{cases}1 & \text { if } x \in W_{1}, \\ a & \text { if } x \in W_{a}, \\ 0 & \text { if } x \in W_{0} .\end{cases}
$$

Clearly $\alpha$ is continuous, preserves both $f$ and $g$ and separates $x$ and $y$.

Now we consider arbitrary $x, y \in X$, with $x \neq y$. By quasi-equation (4) we have $f(x) \neq f(y)$ or $g(x) \neq g(y)$. Without loss of generality we may assume that $f(x) \neq f(y)$. As $f(x), f(y) \in P_{\mathbf{X}}$, by the previous case there exists a map $\alpha \in \mathbf{y}(\mathbf{X}, \underset{\sim}{\mathbf{M}})$ that separates $f(x)$ and $f(y)$. As $\alpha$ preserves $f$, we conclude that $\alpha(x) \neq \alpha(y)$.

Theorem 6.2. Let $\underset{\sim}{\mathbf{M}}=\langle\{0, a, 1\} ; f, g, \mathcal{T}\rangle$ and define $\boldsymbol{X}:=\mathbb{I S}_{\mathrm{c}} \mathbb{P}^{+}(\underset{\sim}{\mathbf{M}})$. Then $\mathbf{X} \in \mathbf{X}$ if and only if $\mathbf{X} \in \mathbf{y}:=\operatorname{Mod}_{\mathcal{T}}(\Sigma)$ and each pair of distinct points of $P_{\mathbf{X}}$ is separated by a clopen downset of $\mathbf{P}_{\mathbf{X}}$. 
Proof. Let $\mathbf{X} \in \boldsymbol{X}$. Certainly, $\mathbf{X} \in \mathbf{y}$. Now let $x, y \in P \mathbf{X}$ with $x \neq y$. Without loss of generality we may assume that $\mathbf{X} \leqslant \mathbf{M}^{S}$ for some non-empty set $S$ and that $x(s)=1$ and $y(s)=0$, for some $s \in S$. Then $\pi_{s}^{-1}(0) \cap P_{\mathbf{X}}$ is a clopen downset in $\mathbf{P}_{\mathbf{X}}$ containing $y$ but not $x$, where $\pi_{s}: M^{S} \rightarrow M$ denotes the $s$ th projection. The converse follows from Lemma 6.1 and the Separation Theorem $([2,1.4 .4])$.

We now deal with the structure ${\underset{\sim}{\sim}}_{h}=\langle\{0, a, 1\} ; f, g, h, \mathcal{T}\rangle$.

Lemma 6.3. Let $\mathbf{X} \in \boldsymbol{y}_{h}$. Then any map $\alpha: X \rightarrow M$ that preserves $f$ and $g$ also preserves $h$.

Proof. Let $x, y \in X$ with $(x, y) \in \operatorname{dom}(h)$. Since $\mathbf{X} \models(5)$, we have $g(x)=f(y)$. As $\alpha$ preserves $f$ and $g$, we obtain

$$
g(\alpha(x))=\alpha(g(x))=\alpha(f(y))=f(\alpha(y)) .
$$

Since $\underset{\sim}{\mathbf{M}}=(5)$, we have $(\alpha(x), \alpha(y)) \in \operatorname{dom}(h)$. Further,

$$
f(h(\alpha(x), \alpha(y)))=f(\alpha(x))=\alpha(f(x))=\alpha(f(h(x, y)))=f(\alpha(h(x, y)))
$$

using the fact that $\mathbf{M} \models(6)$, that $\alpha$ preserves $f$, and that $\mathbf{X} \models(6)$.

Analogously, $g(h(\alpha(x), \alpha(y)))=g(\alpha(h(x, y)))$. Now, by quasi-equation (4), we obtain $\alpha(h(x, y))=h(\alpha(x), \alpha(y))$. Hence $\alpha$ preserves $h$.

Theorem 6.4. Let ${\underset{\sim}{\sim}}_{h}=\langle\{0, a, 1\} ; f, g, h, \mathcal{T}\rangle$ and define $\boldsymbol{X}_{h}:=\mathbb{I S}_{\mathrm{c}} \mathbb{P}^{+}\left(\mathbf{M}_{h}\right)$. Then $\mathbf{X} \in \boldsymbol{X}_{h}$ if and only if $\mathbf{X} \in \mathbf{y}_{h}:=\operatorname{Mod}_{\mathcal{T}}\left(\Sigma_{h}\right)$ and each pair of distinct points of $P_{\mathbf{X}}$ is separated by a clopen downset of $\mathbf{P}_{\mathbf{X}}$.

Proof. Necessity follows exactly as in the proof of Theorem 6.2. To prove sufficiency, let $\mathbf{X} \in \mathbf{y}_{h}$ and assume that each pair of distinct points of $P_{\mathbf{X}}$ is separated by a clopen downset of $\mathbf{P}_{\mathbf{X}}$. By Lemmas 6.1 and 6.3, the morphisms in $\mathbf{y}_{h}\left(\mathbf{X}, \mathbf{M}_{h}\right)$ separate the points of $X$. By the Separation Theorem (1.4.4 in [2]), it remains to show that if $x, y \in X$ with $(x, y) \notin \operatorname{dom}(h)$, then there exists $\alpha \in \mathbf{y}_{h}\left(\mathbf{X}, \mathbf{M}_{h}\right)$ with $(\alpha(x), \alpha(y)) \notin \operatorname{dom}(h)$. Let $x, y \in X$ with $(x, y) \notin \operatorname{dom}(h)$. Then, as $\mathbf{X} \models(5)$, we have $g(x) \neq f(y)$. By Lemmas 6.1 and 6.3 once again, there is a morphism $\alpha \in \boldsymbol{y}_{h}\left(\mathbf{X}, \mathbf{M}_{h}\right)$ such that $\alpha(g(x)) \neq \alpha(f(y))$. This gives $g(\alpha(x)) \neq f(\alpha(y))$, whence $(\alpha(x), \alpha(y)) \notin \operatorname{dom}(h)$, since ${\underset{\sim}{\sim}}_{h} \models(5)$.

Finally, we deal with the structure ${\underset{\sim}{\sim}}_{m}=\langle\{0, a, 1\} ; f, g, m, \mathcal{T}\rangle$. We require some elementary facts about the partial operation $m$ and the relation $\preccurlyeq$.

Lemma 6.5. Let $\mathbf{X} \in \boldsymbol{y}_{m}$.

(a) For all $u, v \in P_{\mathbf{X}}$, we have $u \preccurlyeq v$ if and only if $(u, v) \in \operatorname{dom}(m)$.

(b) $\preccurlyeq$ is an order relation on $P_{\mathbf{X}}$.

(c) Let $u, v \in P_{\mathbf{X}}$. If $(u, v) \in \operatorname{dom}(m)$ and $m(u, v) \in P_{\mathbf{X}}$, then $u=v=m(u, v)$.

(d) Any map $\alpha: X \rightarrow M$ that preserves $f$ and $g$ also preserves $m$. 
Proof. The definition of $\preccurlyeq$ and quasi-equations (7) and (8) give (a). By Lemma 3.1, the relation $\preccurlyeq$ is reflexive and anti-symmetric and, by (a), quasi-equation (9) says precisely that $\preccurlyeq$ is transitive. This proves (b). If $(u, v) \in \operatorname{dom}(m)$ and $m(u, v) \in P_{\mathbf{X}}$, then since $P_{\mathbf{X}}=\operatorname{fix}(f)=f i x(g)$, it follows by quasi-equation (8) that

$$
u=f(m(u, v))=m(u, v)=g(m(u, v))=v .
$$

Thus, (c) holds. To prove (d), assume that the map $\alpha: X \rightarrow M$ preserves $f$ and $g$. Let $(x, y) \in \operatorname{dom}(m)$. By quasi-equation (8), $f(m(x, y))=x$ and $g(m(x, y))=y$. As $\alpha$ preserves $f$ and $g$, we obtain

$f(\alpha(m(x, y)))=\alpha(f(m(x, y)))=\alpha(x)$ and $g(\alpha(m(x, y)))=\alpha(g(m(x, y)))=\alpha(y)$,

so $(\alpha(x), \alpha(y)) \in \operatorname{dom}(m)$ and $m(\alpha(x), \alpha(y))=\alpha(m(x, y))$, by quasi-equation (7).

Recall that the category of Priestley spaces is denoted by $\mathcal{P}$ and that $\mathbf{P}_{\mathbf{X}}$ will be a Priestley space provided that, for all $u, v \in P \mathbf{X}$ with $u \npreceq v$, there exists a clopen downset $U$ such that $v \in U$ and $u \notin U$.

Theorem 6.6. Let $\underset{\sim}{\mathbf{M}_{m}}=\langle\{0, a, 1\} ; f, g, m, \mathcal{T}\rangle$ and define $\boldsymbol{X}_{m}:=\mathbb{I S}_{\mathrm{c}} \mathbb{P}^{+}\left({\underset{\sim}{\sim}}_{m}\right)$. Then $\mathbf{X} \in \boldsymbol{X}_{m}$ if and only if $\mathbf{X} \in \mathbf{y}_{m}:=\operatorname{Mod}_{\mathcal{T}}\left(\Sigma_{m}\right)$ and $\mathbf{P}_{\mathbf{X}}$ is a Priestley space.

Proof. Let $\mathbf{X} \in \boldsymbol{X}_{m}$ and, without loss of generality, assume that $\mathbf{X} \leqslant \mathbf{M}_{m}^{S}$ for some non-empty set $S$. Certainly, $\mathbf{X} \in \mathbf{y}_{m}$. To show that $\mathbf{P}_{\mathbf{X}}$ is a Priestley space, let $x, y \in P_{\mathbf{X}}$ with $x \npreceq y$. Then, by Lemma $6.5(\mathrm{a})$, we have $(x, y) \notin \operatorname{dom}(m)$. This means that there exists $s \in S$ such that $x(s)=1$ and $y(s)=0$. Then $\pi_{s}^{-1}(0) \cap P_{\mathbf{X}}$ is a clopen downset in $\mathbf{P}_{\mathbf{X}}$ containing $y$ but not $x$.

Conversely, let $\mathbf{X} \in \mathbf{y}_{m}$ and assume that $\mathbf{P}_{\mathbf{X}}$ is a Priestley space. Hence, each pair of distinct points of $P_{\mathbf{X}}$ is separated by a clopen downset of $\mathbf{P}_{\mathbf{X}}$. By Lemma 6.1 and Lemma 6.5(d), the morphisms in $\boldsymbol{y}_{m}\left(\mathbf{X}, \mathbf{M}_{m}\right)$ separate the points of $X$. Again, by the Separation Theorem (1.4.4 in [2]), it remains to show that if $x, y \in X$ with $(x, y) \notin \operatorname{dom}(m)$, then there exists $\alpha \in \mathbf{y}_{m}\left(\mathbf{X}, \mathbf{M}_{m}\right)$ with $(\alpha(x), \alpha(y)) \notin \operatorname{dom}(m)$. On this occasion, we shall prove the contrapositive. Let $x, y \in X$ and assume that

$$
\left(\forall \alpha \in \boldsymbol{y}_{m}\left(\mathbf{X},{\underset{\sim}{\sim}}_{m}\right)\right)(\alpha(x), \alpha(y)) \in \operatorname{dom}(m) .
$$

We must prove that $(x, y) \in \operatorname{dom}(m)$. Let $\varphi \in \mathcal{P}\left(\mathbf{P}_{\mathbf{X}}, \mathbf{D}\right)$ and define $\varphi^{*}: X \rightarrow M$ by $\varphi^{*}(x):=m(\varphi(f(x)), \varphi(g(x)))$. Since $\mathbf{X} \models(1)$ it follows, exactly as in the proof of Proposition 3.2, that $\varphi^{*}$ is well defined, continuous and preserves $f$ and $g$. By Lemma $6.5(\mathrm{~d}), \varphi^{*}$ also preserves $m$, whence $\varphi^{*} \in \mathbf{y}_{m}\left(\mathbf{X}, \mathbf{M}_{m}\right)$. Hence, by $(*)$, we have $\left(\varphi^{*}(x), \varphi^{*}(y)\right) \in \operatorname{dom}(m)$ that is,

$$
(m(\varphi(f(x)), \varphi(g(x))), m(\varphi(f(y)), \varphi(g(y)))) \in\{(0,0),(0,1),(1,1)\} .
$$


From this it follows, using Lemma 6.5(c), that

$$
\varphi(f(x))=\varphi(g(x)) \leqslant \varphi(f(y))=\varphi(g(y)) \text { in } \underset{\sim}{\mathbf{D}} .
$$

Since this holds for every $\varphi \in \mathcal{P}\left(\mathbf{P}_{\mathbf{X}}, \underset{\sim}{\mathbf{D}}\right)$, the fact that $\mathbf{P}_{\mathbf{X}}$ is a Priestley space yields

$$
f(x)=g(x) \preccurlyeq f(y)=g(y) \text { in } \mathbf{P}_{\mathbf{X}} .
$$

Hence, since $\mathbf{X} \models(7),(11)$, we have

$$
x=m(f(x), g(x))=f(x) \preccurlyeq f(y)=m(f(y), g(y))=y .
$$

Thus, $x, y \in P_{\mathbf{X}}=\operatorname{fix}(f)$ with $x \preccurlyeq y$, whence $(x, y) \in \operatorname{dom}(m)$, by Lemma 6.5(a).

\section{REFERENCES}

[1] D. M. Clark and B. A. Davey, The quest for strong dualities, J. Austral. Math. Soc. (Series A) $\mathbf{5 8}$ (1995), 248-280.

[2] D. M. Clark and B. A. Davey, Natural Dualities for the Working Algebraist, Cambridge University Press, Cambridge, 1998.

[3] D. M. Clark, B. A. Davey, M. Haviar, J. G. Pitkethly, M. R. Talukder, Standard topological quasi-varieties, Houston J. Math. 29 (2003), 859-887.

[4] D. M. Clark and P. H. Krauss, Topological quasivarieties, Acta Sci. Math. (Szeged) 47 (1984), 3-39.

[5] B. A. Davey, Duality theory on ten dollars a day, Algebras and Orders (I. G. Rosenberg and G. Sabidussi, eds), NATO Advanced Study Institute Series, Series C, Vol. 389, Kluwer Academic Publishers, 1993, pp. 71-111.

[6] B. A. Davey, Dualisability in general and endodualisability in particular, Logic and Algebra (Pontignano, 1994), (A. Ursini and P. Aglianò, eds), Lecture Notes in Pure and Appl. Math. 180, Dekker, New York, 1996, pp. 437-455.

[7] B. A. Davey and M. Haviar, A schizophrenic operation which aids the efficient transfer of strong dualitites, Houston Math. J. 26 (2000), 215-222.

[8] B. A. Davey, M. Haviar and H. A. Priestley, Endoprimal distributive lattices are endodualisable, Algebra Universalis 34 (1995), 444-453.

[9] B. A. Davey, M. Haviar and H. A. Priestley, The syntax and semantics of entailment in duality theory, J. Symbolic Logic 60 (1995), 1087-1114.

[10] B. A. Davey, M. Haviar and R. Willard, Structural entailment, Algebra Universalis (to appear).

[11] B. A. Davey and J. G. Pitkethly, Endoprimal algebras, Algebra Universalis, 38, (1997), 266-288.

[12] B. A. Davey and H. A. Priestley, Introduction to Lattices and Order, second edition, Cambridge University Press, Cambridge, 2002.

[13] B. A. Davey and H. Werner, Dualities and equivalences for varieties of algebras, Contributions to Lattice Theory (Szeged, 1980), (A. P. Huhn and E. T. Schmidt, eds) Coll. Math. Soc. János Bolyai 33, North-Holland, Amsterdam, 1983, pp. 101-275. 
[14] H. A. Priestley, Representation of distributive lattices by means of ordered Stone spaces, Bull. London Math. Soc. 2 (1972), 186-190.

[15] A. Stralka, A partially ordered space which is not a Priestley space, Semigroup Forum 20 (1980), 293-297.

Brian A. DAVEy

Department of Mathematics, La Trobe University, Victoria 3086, Australia e-mail: B.Davey@latrobe.edu.au

Miroslav Haviar

Department of Mathematics, Matej Bel University, PdF, Ruzova 13, 97411 Banska Bystrica, Slovak Republic

e-mail: haviar@pdf.umb.sk

Ross WiLLARD

Department of Pure Mathematics, University of Waterloo, Waterloo, Ontario N2L 3G1, Canada

e-mail: rdwillar@uwaterloo.ca

(1) To access this journal online:

(20) http://www.birkhauser.ch 